\title{
Chemical Probes Unravel an Antimicrobial Defense Response Triggered by Binding of the Human Opioid Dynorphin to a Bacterial Sensor Kinase
}

\author{
Megan H. Wright, ${ }^{* \dagger}{ }^{\dagger}$ Christian Fetzer, ${ }^{(i)}$ and Stephan A. Sieber*(i) \\ Department of Chemistry, Technical University of Munich, Lichtenbergstr. 4, D-85748 Garching, Germany \\ Supporting Information
}

ABSTRACT: Host-microbe communication via small molecule signals is important for both symbiotic and pathogenic relationships, but is often poorly understood at the molecular level. Under conditions of host stress, levels of the human opioid peptide dynorphin are elevated, triggering virulence in the opportunistic pathogenic bacterium Pseudomonas aeruginosa via an unknown pathway. Here we apply a multilayered chemical biology strategy to unravel the mode of action of this putative interkingdom signal. We designed and applied dynorphin-inspired photoaffinity probes to reveal the protein targets of the peptide in live bacteria via chemical proteomics. ParS, a largely uncharacterized membrane sensor of a twocomponent system, was identified as the most promising hit.

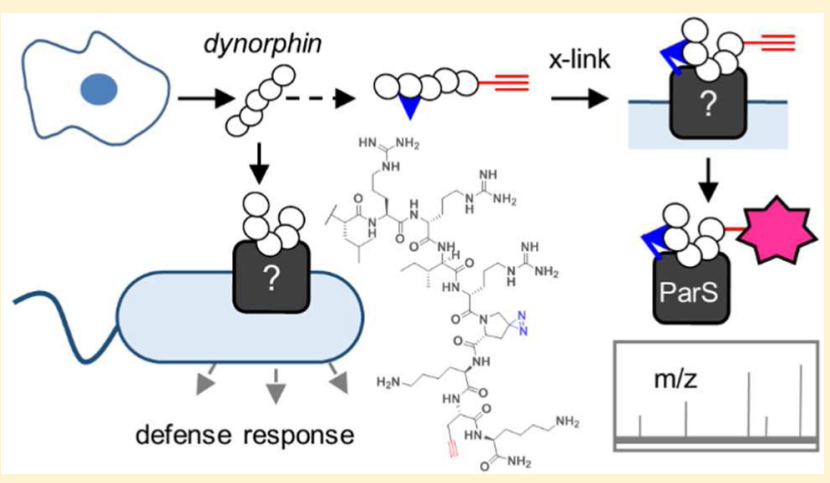
Subsequent full proteome studies revealed that dynorphin(1-13) induces an antimicrobial peptide-like response in Pseudomonas, with specific upregulation of membrane defense mechanisms. No such response was observed in a parS mutant, which was more susceptible to dynorphin-induced toxicity. Thus, $P$. aeruginosa exploits the ParS sensing machinery to defend itself against the host in response to dynorphin as a signal. This study highlights interkingdom communication as a potential essential strategy not only for induction of $P$. aeruginosa virulence but also for maintaining viability in the hostile environment of the host.

\section{INTRODUCTION}

The dynorphins are important endogenous human peptide hormones that act primarily through binding to the $\kappa$-opioid receptor (OPRK) and have roles in pain response, stress, and addiction, among others. ${ }^{1}$ Opioids and their receptors are important components of the peripheral nervous system, but they also accumulate during host stress at sites of inflammation. ${ }^{2}$ Coupled with evidence that bacteria activate virulence in response to host stress, ${ }^{3}$ these observations suggest that opioids may be recognized by bacteria as signaling molecules. A small molecule OPRK agonist and the peptide hormone dynorphin-A (Dyn-A) were shown to activate quorum sensing and result in enhanced virulence of the opportunistic pathogen Pseudomonas aeruginosa $(P A) .{ }^{4} P A$ is one of the most commonly isolated microbes in patients with bloodstream and lung infections in acute care settings in Europe and the leading cause of death in patients with cystic fibrosis. ${ }^{5}$ The pathogen is also very challenging to treat due to its high intrinsic resistance to antibiotics and its ability to modulate its behavior in widely different environments and in response to a host. This ability may stem in part from the organism's large number of two-component sensors, its large genome, and its complex quorum sensing networks. ${ }^{6}$ In a rodent stress model, Dyn-A was shown to be released in the intestine where it bound to and was taken up by bacteria. ${ }^{4} \mathrm{~A}$ subsequent study showed that $P A$ regulates its response to a small molecule opioid depending on the abundance of nutrients in the environment, integrating multiple inputs in the decision to switch to the costly virulence phenotype. ${ }^{7}$ Bacteria have receptors that recognize other host molecules (e.g., interferon$\gamma^{8}$ and adrenergic hormones $\left.{ }^{9}\right)$. However, the putative dynorphin receptor and the majority of the response circuitries mediating bacterial response to opioids are completely unknown (Figure 1a). Targeting virulence or manipulating host-microbe or microbe-microbe communications have been put forward as possible nontraditional approaches to tackle the rise of multidrug-resistant bacteria. However, the pathways and environmental conditions, such as host stress and interkingdom signaling, that lead to microbial virulence are poorly explored.

Here, we use a chemical biology approach to unravel the mechanism of action of the human opioid peptide Dyn-A in $P A$. Phenotypic studies first confirm that the virulence-inducing effect of a truncated dynorphin analogue (DYN) is peptide sequence dependent. We then employ a chemical proteomic approach using DYN-inspired photoaffinity probes to identify the membrane sensor ParS as a putative binding partner of DYN in live bacteria. In-depth global proteomic analyses further define the specific defense response evoked in $P A$ by DYN. Finally, we show that this defense response is abrogated

Received: January 31, 2017

Published: March 28, 2017 


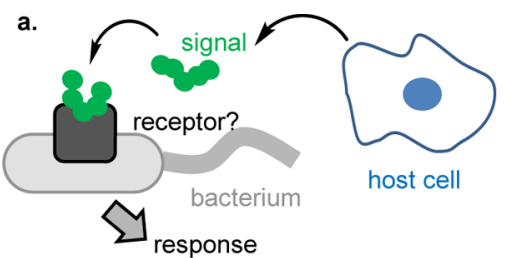

b. DYN Y G G F L R R I R P K L K - NH SCR L F K Y R R I G P R G K L $-\mathrm{NH}_{2}$ CC L S I A T R H Q R V R V K $-\mathrm{NH}_{2}$
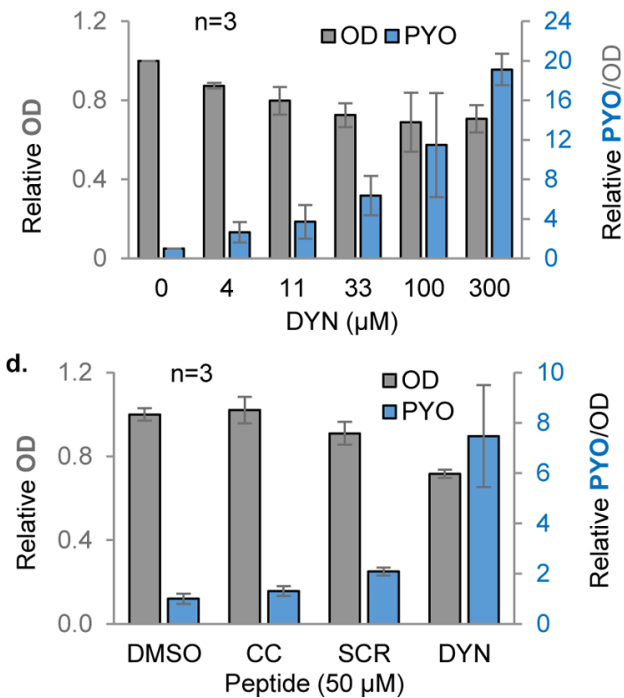

Figure 1. DYN induces virulence in Pseudomonas aeruginosa in a sequence-specific manner. (a) Dynorphin is a human peptide hormone released by host cells during stress. It triggers a virulence response in the bacterium $P A$ via an unknown pathway and putative receptor. (b) Sequences of DYN and control peptides. (c) The effect of DYN on growth, as measured by optical density (OD, gray bars), and on the production of the virulence factor pyocyanin (PYO, blue bars) in PAO1. Error bars show standard deviation from three independent biological experiments. OD and PYO were normalized to DMSO control in each case. (d) Analyzing the sequence specificity of the DYN response using the peptides shown in (b).

in a mutant lacking ParS, functionally linking peptide binding to ParS with the phenotypic response.

\section{RESULTS}

Dynorphin and Derivatives Induce Pseudomonas Virulence. Since $P A$ virulence is known to be highly environment dependent, we first sought to define the conditions under which dynorphin induced a response. Previous work has linked the peptide Dyn-A (dynorphin(117)-OH) and the small molecule opioid U50,488 to induction of virulence in $P A$ strain PAO1., ${ }^{4,7}$ We confirmed that in our hands these compounds also induced the production of the blue-green toxin pyocyanin under similar conditions of nutrient limitation, as did a truncated Dyn-A analogue with a C-terminal amide (DYN, dynorphin $(1-13)-\mathrm{NH}_{2}$; Figure S1). The $\mathrm{N}$ terminus of dynorphin is critical for signaling via human opioid receptors, and this truncated peptide is equipotent in the human system. ${ }^{10}$ However, although they induced a similar response to each other, the two peptides behaved quite differently compared to the small molecule: There was significant growth reduction at higher concentrations of peptide and a corresponding drop-off of pyocyanin production, whereas U50,488 was less toxic to bacteria and only induced pyocyanin at higher concentrations (Figure S1). It is therefore not clear that dynorphin-based peptides and U50,488 have the same phenotype and thus mode of action. We decided to focus on the endogenous peptide signals and, given the similar responses of the two peptides, chose the shorter dynorphin analogue, DYN, for synthetic tractability. We used a chemically defined MOPS-buffered succinate medium to optimize the iron and phosphate contents, since these components are important for the virulence responses of $P A$ (Figure S1). ${ }^{7}$ Our optimized conditions generated more reproducible data, and we observed that DYN (Figure $1 \mathrm{~b}$ ) induced mild toxicity and production of pyocyanin in a dose-dependent manner (Figure 1c).

To establish whether the sequence of DYN is important for the observed phenotype, we tested a sequence-scrambled peptide (SCR) and a charge-control peptide (CC), which has a different composition but is still highly basic (Figure $1 \mathrm{~b}$ ). The charge control showed no significant effect on either growth or virulence, and the scrambled peptide a greatly reduced effect (Figure 1d), suggesting a highly specific response to DYN.

Photoaffinity Profiling of Dynorphin Targets. Intrigued by this phenotype, we next sought to identify protein interacting partners of DYN in live bacteria using photoprobes. Photoaffinity labeling is a technique used to explore small molecule-protein binding. ${ }^{11,12}$ A chemical probe mimicking the molecule of interest is equipped with a photoreactive group and a label or tag for detection. The probe is incubated with lysates or live cells, and, upon UV irradiation, a highly reactive intermediate is generated from the photoreactive group which cross-links the probe to its biomolecular binding partners (Figure 2a). Common photoreactive groups include benzophenone (Bpa), aryl azides $\left(\mathrm{ArN}_{3}\right)$, and diazirines (Di) (Figure 2b), which possess different photoproperties and steric footprints. ${ }^{13}$ Given their distinct and complementary properties, it is difficult to predict which photogroup will perform best in any one system; therefore we investigated all three. Rather than modify the peptide with a large label for detection, we incorporated a terminal alkyne into the structure of probes; this pretag allows subsequent attachment of a fluorophore or biotin affinity label via copper-catalyzed azide-alkyne cycloaddition (CuAAC) for enrichment and visualization (Figure 2a). Probes were synthesized using Fmoc-SPPS chemistry. The bulkiest photogroup, Bpa, was incorporated at two different positions: in place of phenylalanine (probe DYN1) and at the C-terminus of the sequence (DYN3; Figure 2b).

Similarly, an aryl azide moiety was incorporated in place of phenylalanine in DYN4 and diazirine-containing "photoproline"14 in place of proline in DYN5. A synthetically facile orthogonal protection strategy also yielded probe DYN9, where a short diazirine building block was coupled to a lysine side chain. Probes DYN5, DYN4, DYN3, and DYN1 all triggered a clear virulence response in $P A$, while the scrambled Bpa probe SCR1 showed very little effect, as anticipated (Figures 3a and S2). Unexpectedly, DYN9 had little effect on pyocyanin production (Figure S2), possibly indicating high sequence sensitivity of the virulence response.

To perform labeling experiments in live cells, $P A$ was grown to early stationary phase, resuspended in PBS, and incubated with probes at $4 \mu \mathrm{M}$ (the onset of virulence induction), and samples were irradiated. Cells were washed and lysed, and lysates subject to $\mathrm{CuAAC}$ to attach a rhodamine fluorophore. 
a.

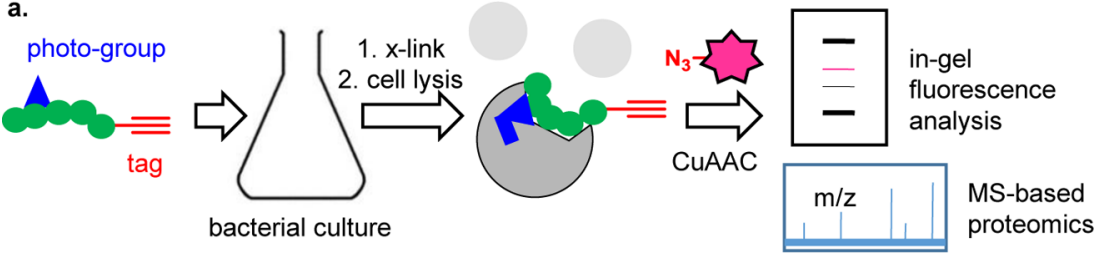

b.

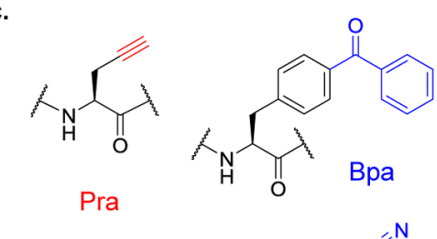

\begin{tabular}{lccccccccccccccc} 
Residue & 1 & 2 & 3 & 4 & 5 & 6 & 7 & 8 & 9 & 10 & 11 & 12 & 13 & 14 & 15 \\
\hline DYN & Tyr & Gly & Gly & Phe & Leu & Arg & Arg & Ile & Arg & Pro & Lys & Leu & Lys & & \\
DYN1 & Tyr & Gly & Gly & Bpa & Leu & Arg & Arg & Ile & Arg & Pro & Lys & Leu & Lys & Pra & \\
DYN3 & Tyr & Gly & Gly & Phe & Leu & Arg & Arg & Ile & Arg & Pro & Lys & Leu & Lys & Bpa & Pra \\
SCR1 & Pra & Bpa & Lys & Tyr & Arg & Arg & Ile & Gly & Pro & Arg & Gly & Lys & Leu & & \\
DYN4 & Tyr & Gly & Gly & ArN $_{3}$ & Leu & Arg & Arg & Ile & Arg & Pro & Lys & Pra & Lys & & \\
DYN5 & Tyr & Gly & Gly & Phe & Leu & Arg & Arg & Ile & Arg & P(Di) & Lys & Pra & Lys & & \\
DYN9 & Tyr & Gly & Gly & Phe & Leu & Arg & Arg & Ile & K(Di) & Pro & Lys & Pra & Lys & &
\end{tabular}
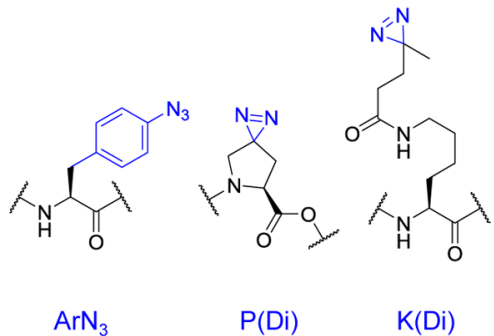

DYN5

Tyr Gly Gly Phe Leu Arg Arg lle K(Di) Pro Lys Pra Lys

$\mathrm{ArN}_{3}$

$\mathrm{P}(\mathrm{Di})$

$\mathrm{K}(\mathrm{Di})$

Figure 2. Design of photoreactive probes mimicking DYN. (a) Workflow for identifying putative DYN protein interactors via chemical proteomics. Probes are incubated with bacterial cultures, and the cultures are irradiated with UV light, thereby photo-cross-linking the probe to its protein binding partners. Probes contain a clickable alkyne for ligation to azide-functionalized fluorophores (for visualization by in-gel fluorescence) or biotin (for affinity enrichment and gel-free LC-MS/MS analysis of tryptic peptides). (b) Sequences of DYN and probes bearing different photo-crosslinkers (all C-terminal amides). $\mathrm{Bpa}=$ benzophenone; $\mathrm{ArN}_{3}=$ aryl azide; $\mathrm{P}(\mathrm{Di})=$ photoproline (diazirine-bearing proline); $\mathrm{K}(\mathrm{Di})=$ diazirinemodified lysine; Pra: propargyl-glycine. (c) Structures of the unnatural amino acid residues.

Separation of proteins by SDS-PAGE and fluorescence scanning revealed some striking differences between probes bearing different photoreactive groups (Figure $3 \mathrm{~b}$ ). All probes exhibited irradiation-dependent labeling, but while aryl azide probe DYN4 labeled distinct bands, both diazirine and Bpa probes additionally gave rise to diffuse bands and a "laddering" pattern, reminiscent of heterogeneous glycolipids. Lipopolysaccharide (LPS) forms a large part of the surface coat of $P A$ and other Gram-negative bacteria and consists of lipid A, a core oligosaccharide, and a variable $\mathrm{O}$-antigen polysaccharide side chain. ${ }^{15}$ Treatment of samples after CuAAC with a protease, proteinase $\mathrm{K}$, indeed showed that the diffuse labeling and laddered bands were not protein (Figure 3c). Furthermore, isolated PA LPS labeled with probes generated a similar pattern (Figures $3 \mathrm{~d}$ and $\mathrm{S} 2$ ). The DYN probes are positively charged at physiological $\mathrm{pH}$, and charge-charge interactions may mediate binding to the negatively charged phosphates of LPS. Supportive of this hypothesis, a scrambled Bpa-containing probe (SCR1, Figure 2b), designed based on the sequence of the scrambled control peptide SCR, gave a similar LPSdominated labeling pattern (Figure $3 \mathrm{~d}$ ).

We initially focused on the Bpa probe DYN1, since probes with this photogroup gave the strongest labeling and high pyocyanin induction in $P A$. Proteins were subject to CuAAC with biotin-azide, enriched on avidin beads, and digested. Peptides from control (DMSO vehicle treated) or DYN1 samples were isotopically dimethyl labeled ${ }^{16}$ for quantification, mixed, and analyzed by gel-free LC-MS/MS. Consistent with the high level of labeling observed on-gel, large numbers of proteins were apparently enriched in DYN1 over control samples, suggestive of high nonspecific binding or photoreactivity (Figure S3). ${ }^{17-19}$

We next performed chemical proteomic analyses with DYN4 and using a similar workflow but label-free $\mathrm{MS}^{1}$ intensity-based quantification (MaxLFQ), ${ }^{20}$ which enables comparison across more samples. We included a competition experiment where bacteria were first incubated with 5-fold excess DYN and then with DYN4, with the aim of differentiating between genuine DYN binders over high abundance or false positive hits. DYN4 was more specific than DYN1 but still gave a large number of potential hits (Figure S3 and Table S1). Outer membrane proteins (OMPs) figured prominently, and biochemical separation of inner and outer membranes supported this result (Figure S4). We also observed a surprising enrichment of ribosomal proteins (Table S1). To check for effects of DYN on protein synthesis, we treated early log-phase bacteria with DYN at $10 \mu \mathrm{M}$ for $2 \mathrm{~h}$ and then incubated aliquots for $30 \mathrm{~min}$ with the methionine analogue azidohomoalanine (AHA), which is incorporated into newly synthesized proteins. ${ }^{21,22}$ Although total protein yield was lower for the DYN-treated bacteria due to reduction in growth, the rate of protein synthesis as measured by pulsed incorporation of AHA was the same (Figure S4). Furthermore, a simple aryl azide "minimal" probe $^{19}$ labeled proteins in the soluble fraction in a similar pattern to DYN4 (Figure S4). Together these data suggest that aryl azide probes exhibit a high degree of nonspecific binding in this system. Lack of LPS labeling by DYN4 (Figure S4) also suggested that this probe is biased in its reactivity. This may be related to the propensity of aryl azides to undergo ring expansion upon irradiation. ${ }^{13}$

Finally, to investigate the protein targets of diazirine probe DYN5, bacteria were incubated with DYN at varying concentrations followed by DYN5 (ratios of 1:1, 1:2, and 1:5 of DYN5:DYN) to generate competition data. Enrichment and label-free proteomics were carried out as before. Analysis of the data revealed more selective labeling compared to DYN1 and DYN4 (Figure 4a), as expected given the lower intensity of ingel labeling. A subset of enriched proteins was significantly outcompeted at 5-fold excess DYN (Figure S5), and three proteins were also outcompeted at 2-fold excess DYN (Figure $4 \mathrm{~b}$ and Table S2). Of these, the protein ParS was alone in being completely absent from DMSO controls. ParS was also detected in DYN4 labeling, although only quantified in two out of three replicates. We analyzed the competition response profiles (LFQ intensity, raw intensity and sequence coverage) of all possible hits (Figures S5 and 4c); ParS showed the most pronounced dose-dependent response.

ParS is a putative membrane sensor kinase and is predicted to be co-expressed with response regulator ParR in a twocomponent system. ${ }^{23}$ The ParRS system has been linked to the response of $P A$ to cationic antimicrobial peptides (AMPs). ${ }^{24}$ AMPs include endogenous host peptides involved in the 


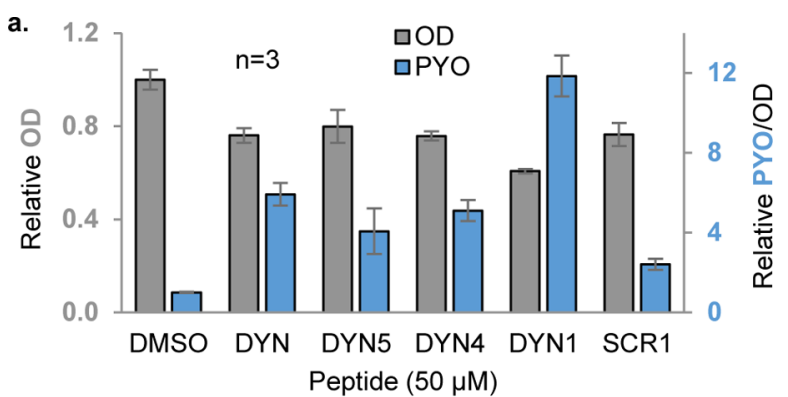

b.

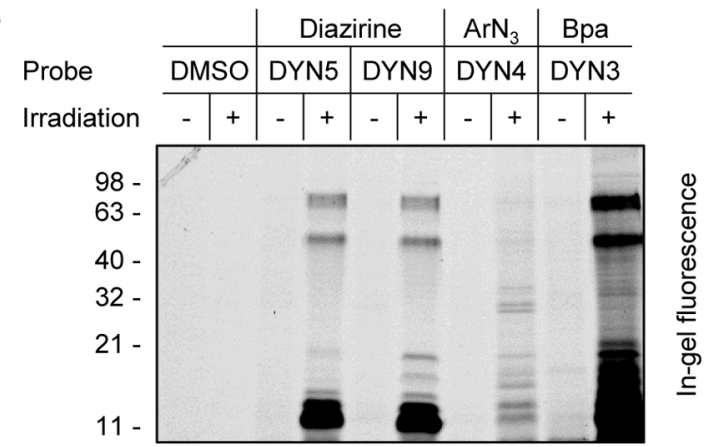

c.

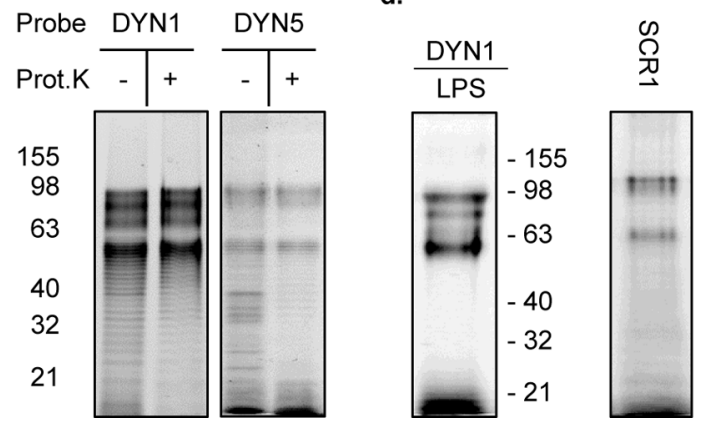

Figure 3. Photoaffinity labeling in live bacteria. (a) Determination of growth (OD, gray) and PYO (blue) induction by probes. Error bars show standard deviation from 3 biological replicates. (b) Gel-based analysis after photolabeling with probes (DYN5, 9, 4, and 3; see Figure 2 ) in live $P A$. Probes were incubated at $5 \mu \mathrm{M}$. Irradiation with UV was performed as indicated; after lysis and separation into soluble (PBS) and insoluble fractions, proteins were subject to CuAAC with rhodamine-azide ( $\mathrm{RhN} 3)$, precipitated with acetone, separated by gel, and imaged for fluorescence. Insoluble fraction shown here. (c) Analysis of probe labeling of bacterial lipopolysaccharide (LPS). Bacteria were labeled with DYN1 or DYN5 and lysed in $0.2 \%$ TX100 in PBS. Following $\mathrm{CuAAC}$ with $\mathrm{RhN} 3$ and precipitation, samples were resuspended, split into two portions, and treated with proteinase $\mathrm{K}$ (Prot.K) or buffer (control) to digest proteinaceous material. Samples were separated on SDS PAGE, and gels fluorescent scanned. (d) Left: probe DYN1 labeling of purified LPS. Right: Scrambled Bpa probe SCR1 labeling of bacteria (as for c). See full gels and Coomassie staining in Figure S2.

immune defense against infection and synthetic compounds in clinical use. Although in many cases their mode of action is unclear, AMPs often bind to the bacterial membrane and rapidly kill cells through formation of pores or membrane disruption. $^{25}$ Select peptides appear to act via ParRS to induce adaptive resistance to other AMPs-a response where the bacterial cell activates specific mechanisms that render it temporarily more resistant to attack. ${ }^{24}$ Although DYN only slightly reduced growth (Figure 1c), it is cationic. Thus, we selected ParS out of the list of possible hits (Figure 4d) as the most promising protein for further analysis.

Total Proteome Analysis of DYN-Treated Pseudomonas Reveals an Antimicrobial Peptide-Like Defense Response. If DYN binds ParS to induce an AMP-like response in $P A$, changes in the expression of specific proteins and pathways should be detectable. We therefore undertook a global proteome analysis of bacteria treated with the peptide. Bacteria were grown for $16 \mathrm{~h}$ in the presence of $10 \mu \mathrm{M}$ DYN (a concentration resulting in a small drop in growth and a measurable increase in pyocyanin; Figure S6).

Cells were subsequently lysed, lysates normalized based on total protein amount, and samples prepared for analysis using the FASP protocol (filter-aided sample preparation), which enables lysis of samples in buffers containing high detergent concentrations to maximize solubilization of membrane proteins. $^{26}$ Peptides were analyzed by LC-MS/MS and quantified by MaxLFQ. ${ }^{20}$ Over 3000 proteins were quantified in at least three biological replicates, with high reproducibility (Pearson correlations of $0.96-0.99$, Figure S6). We observed a specific response, with only $5.5 \%$ of proteins significantly affected by DYN treatment ( $t$-test; Figure $5 a$ and Table S3). Gene ontology analysis suggested that siderophore biosynthesis and transport, sulfur compound metabolism, localization, and transport processes were overrepresented among downregulated proteins, indicating moderate changes in iron homeostasis and uptake (Figure S7). Proteins upregulated by DYN treatment included those involved in lipid A biosynthesis and belong to the arn operon (ArnA, B, C, D, and T; Figures 5 and S8), which is upregulated in response to diverse AMPs. ArnT adds 4-aminoarabinose to lipid A phosphate groups, reducing the negative charge of the membrane and promoting resistance to AMPs. ${ }^{15}$ The arn operon is under the control of various twocomponent systems in $P A$, including ParRS, PmrAB, and CprRS. $^{24,27,28}$ pmrA and $p m r B$ are also upregulated by AMPs, ${ }^{27}$ and expression levels of these two proteins increased strongly in response to DYN in our analysis.

Another highly upregulated protein with a known role in enhanced AMP resistance was SpeE2 (PA4774), ${ }^{29}$ which, together with another upregulated product (PA4775), is predicted to be in the same operon as $p m r A B$. Another set of proteins that responded to DYN belong to the resistancenodulation-division (RND) family and have known or putative roles in drug efflux. These tripartite complexes consist of an RND transporter, a periplasmic membrane fusion protein, and an outer membrane factor and act to pump antibiotics across the cell membrane. ${ }^{30,31}$ All proteins from the mexGHI-opmD operon (PA4205-8) were significantly upregulated, as were MexX and MexY (PA2018-19; Table S3). In contrast, the porin OprD, annotated as a basic amino acid, basic peptide, and imipenem outer membrane transporter, was downregulated. Interestingly, the ParRS system has been previously linked to these changes. ${ }^{32}$

Another virulence-related protein, PA-I galactophilic lectin (LecA) was strongly upregulated in our data. LecA is a cytotoxic lectin and adhesion molecule implicated in biofilm formation $^{33}$ and induced in response to another host signal, interferon $-\gamma .{ }^{8}$ The data described above were collected incubation of DYN throughout growth until stationary phase. To explore the effects of transient DYN exposure, bacteria were grown to early exponential phase and then incubated with a slightly lower concentration of $5 \mu \mathrm{M}$ DYN (to limit the mild inhibition of growth that DYN induces), and the global 


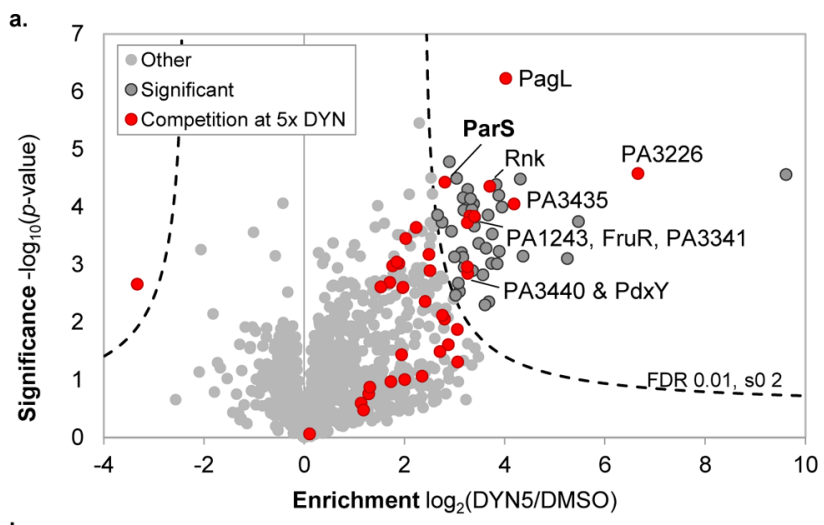

b.

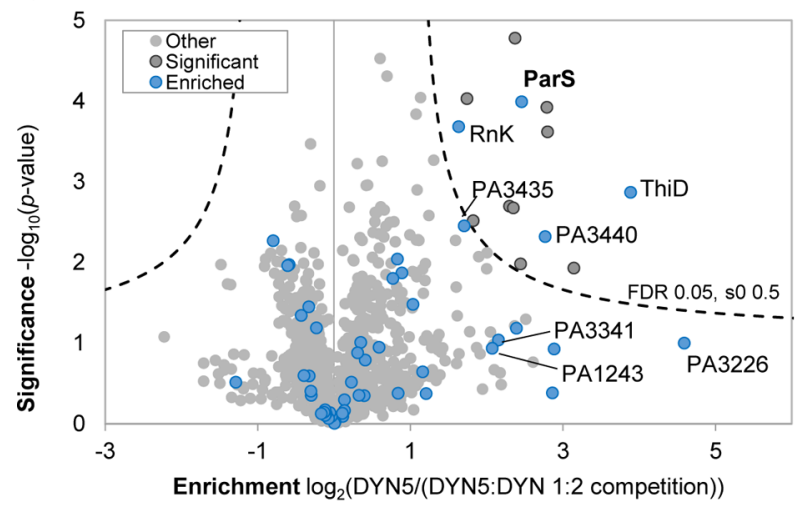

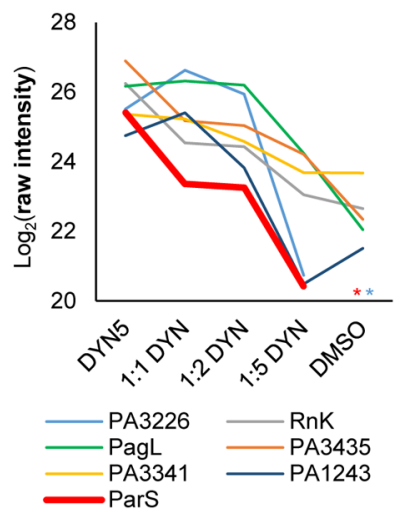

d.

\begin{tabular}{|l|l|l|}
\hline Uniprot & Gene & Protein \\
\hline Q9HYQ4 & PA3341 & $\begin{array}{l}\text { transcriptional } \\
\text { regulator }\end{array}$ \\
\hline Q9HZ14 & PA3226 & hydrolase \\
\hline Q9HTS6 & PA5274 & $\begin{array}{l}\text { Nucleoside } \\
\text { diphosphate kinase } \\
\text { regulator (Rnk) }\end{array}$ \\
\hline Q9HYH1 & PA3435 & $\begin{array}{l}\text { Uncharacterized } \\
\text { (oxidoreductase) }\end{array}$ \\
\hline Q9HVD1 & PA4661 & $\begin{array}{l}\text { Lipid A deacylase } \\
\text { PagL }\end{array}$ \\
\hline Q91495 & PA1243 & $\begin{array}{l}\text { sensor/response } \\
\text { regulator hybrid }\end{array}$ \\
\hline Q912U4 & PA1798 & $\begin{array}{l}\text { Two-component } \\
\text { sensor, ParS }\end{array}$ \\
\hline
\end{tabular}

Figure 4. Chemical proteomics with diazirine probe DYN5 reveals ParS as a potential target. Bacteria were incubated with $3 \mu$ M DYN5, or DYN5 plus increasing concentrations of DYN $\left(1,2\right.$, or 5-fold), or DMSO control, irradiated, lysed, proteins ligated by CuAAC to biotin- $\mathrm{N}_{3}$, enriched on avidin beads, digested by trypsin, and peptides analyzed by LC-MS/MS. Three independent biological replicate labeling experiments were performed on different days. Data were analyzed by MaxLFQ, filtered to retain only proteins identified by multiple peptides, and quantified in $2 / 3$ biological replicates, and missing values were imputed to mimic noise. Two-sample permutation-corrected $t$-tests with FDR (false discovery rate) control were performed to compare samples. (a) Volcano plot depicting enrichment and significance of enrichment in chemical proteomics experiments with 3 $\mu \mathrm{M}$ DYN5. In red: proteins shown to be outcompeted by $5 \times$ excess DYN (see Figure S5); $n=3$ biological replicates. (b) Volcano plot showing results of competition between DYN5 and $2 \times$ excess DYN. In blue: proteins enriched over DMSO controls (see a). (c) Profile plots of potential hits (raw MS ${ }^{1}$ intensity). * = value of zero in DMSO control for these proteins. Proteins selected showed a dose-response (see Figure S5), significant enrichment over DMSO controls, and significant out-competition by DYN. FruR, PA33440, and PdxY did not show a consistent dose-response. (d) The most promising hits based on competition and enrichment and their putative roles, see Table S2.

proteome analyzed (Table S4). Only $0.75 \%$ of proteins showed a significant response under these conditions: Again, proteins in the arn and $p m r A B$ operons were the most prominent upregulated hits (Figure $5 \mathrm{~b}$ ). Thus, arn and $p m r A B$ upregulation is a rapid, direct response to DYN under different growth stages.

In all analyses, ParR and ParS proteins themselves were unaffected by DYN treatment; however, this is not inconsistent with their role as mediators since a previous study by Fernández et al. reported that the AMP indolicidin had no effect on parRS expression, although this peptide triggered responses via parRS. We compared our data set to the microarray transcriptome data of Fernández et al. ${ }^{24}$ Proteins in the arn and pmr operons, significantly upregulated by DYN treatment in our analysis, were indeed induced by indolicidin treatment in wild-type relative to a parR mutant (Table S6).

ParS Mediates the DYN-Induced Phenotype. We hypothesized that of the possible DYN targets (Figure 4d), ParS was most likely responsible for the AMP-defense phenotype. To test this hypothesis more directly, a PAO1 parS transposon mutant was obtained (PA Two-Allele Library, University of Washington), validated by PCR (Figure S9), and assayed for response to DYN. Both this and a parR mutant were dramatically more susceptible to DYN-induced toxicity than the original wild-type (wt) strain, and we did not observe any pyocyanin production in response to the peptide at nontoxic concentrations (Figure S9). Using a low DYN concentration of $1 \mu \mathrm{M}$ to minimize effects on growth, we repeated the global proteome analysis of the parS mutant or wt $P A$ treated with DYN for $16 \mathrm{~h}$ (Figure S6 and Table S5). Even at this low concentration, there was strong upregulation of Arn proteins and PmrA \& B in wt bacteria, while cells lacking ParS showed no significant response at all (Figure 6b). Although there are differences between the parS mutant and wt strain proteomes, most proteins altered by DYN treatment in the wt were expressed at comparable levels (Figure S10). Furthermore, although parR and parS mutants were more susceptible to DYN treatment (Figure $6 \mathrm{~b}$ ), other strains from the library, such as a strain mutant in the unrelated response regulator phoP, showed a similar growth and phenotypic response to the wt (Figure S9). Together these data strongly link ParS to the AMP-defense response of Pseudomonas to DYN.

\section{DISCUSSION}

Here we use chemical probes to identify a membrane sensor kinase, ParS, as a binding partner of the human peptide dynorphin and the mediator of a highly specific bacterial defense response. 

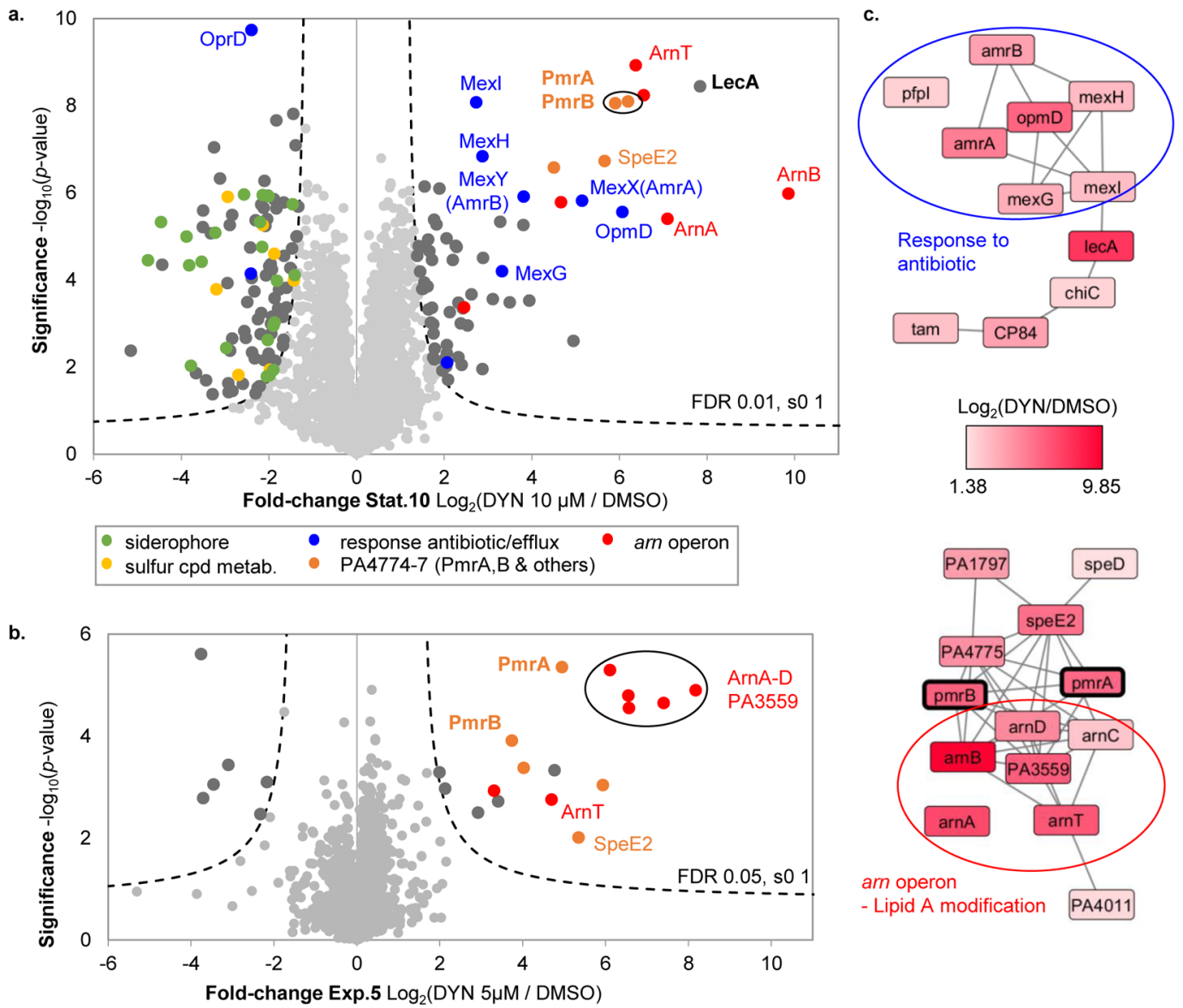

Figure 5. Global proteomics reveals specific changes in response to DYN. (a) Volcano plot visualization of global proteomics experiment "Stat.10". $P A$ was grown in the presence of DYN at $10 \mu \mathrm{M}$ for $16 \mathrm{~h}$ until stationary phase. Bacteria were harvested and lysed and samples prepared by FASP for LC-MS/MS. Quantification was carried out with MaxLFQ; $n=5$ (biological replicates). After filtering to discard proteins not identified in at least four replicates, missing values were imputed, and a two-sample $t$-test (permutation-based, FDR 0.01, s0 1) carried out to compare DMSO and DYN treated samples. Proteins of particular interest are labeled and color-coded by putative function/pathway, see also Table S3. (b) Global proteomicsexponential phase, data set "Exp.5". PA was grown to OD 0.2-0.3 then incubated with DYN at $5 \mu \mathrm{M}$ for $2 \mathrm{~h}$ during exponential growth. Samples were analyzed as above; $n=3$ (replicates grown from independent starter cultures). Only proteins identified in at least duplicate were used in analysis. Missing values were imputed (downshift 1.8, width 0.3), and a two-sample $t$-test (permutation-based, FDR 0.05 , s0 1) carried out to compare DMSO and DYN treated samples, see also Table S4. (c) Cytoscape analysis of upregulated proteins (from a; see also Figure S8). Edges between nodes represent predicted functional or physical interactions. Analysis of downregulated proteins is shown in Figure S7.

Dynorphin $(1-17)$ and the shorter $(1-13)$ analogue (DYN) both induced production of the virulence factor pyocyanin. Interestingly this phenotype was highly medium dependent, indicating that $P A$ integrates multiple environmental signals in its response to these compounds. Pyocyanin induction was also peptide sequence dependent; however, binding of DYN-based photoaffinity probes to the LPS was subsequently shown to be sequence independent, suggesting that LPS interaction is not responsible for induction of virulence but may be important for initial charge-mediated coordination with the cell membrane. Photoaffinity probes with different photoreactive groups exhibited very distinct profiles, and the lack of specificity exhibited by both benzophenones and aryl azides, as noted recently by us and others, ${ }^{17,19}$ led us to focus on diazirinecontaining probes. We identified the membrane protein ParS, the sensing partner of a two-component response system, as a promising hit.

Subsequent in-depth global proteome analyses of bacteria treated with DYN at different growth stages revealed a highly specific, parS-dependent, response: A dramatic increase in the levels of proteins that boost membrane defense mechanisms against cationic peptides. Upregulated proteins included those in the arn operon that modify lipid A, reducing the negative charge of the membrane and thereby increasing AMP resistance. Such modifications also alter outer membrane permeability and host immune recognition of LPS and are common in clinical isolates of $P A$ from cystic fibrosis patients. ${ }^{34}$ These data are consistent with previous reports of parRSdependent arn upregulation and adaptive AMP resistance. ${ }^{24}$ Previous work showed that ParRS and a second twocomponent system CprRS mediate the response to diverse AMPs in $P A$ in a peptide-dependent manner. ${ }^{28}$ CprRS was not detected in our analyses, suggesting that it is not involved in DYN-dependent signaling. Interestingly, DYN is only weakly toxic but induces strong upregulation of defense pathways, consistent with previous data indicating that the signaling and growth inhibition functions of AMPs are not directly coupled. ${ }^{24,27,28}$ More work is needed to understand the structure-activity relationships of AMP signaling and the exact binding site of the peptides, but it is tempting to speculate that DYN binds at the predicted negatively charged periplasmic loops of ParS, in a similar manner to peptide binding to sensor 


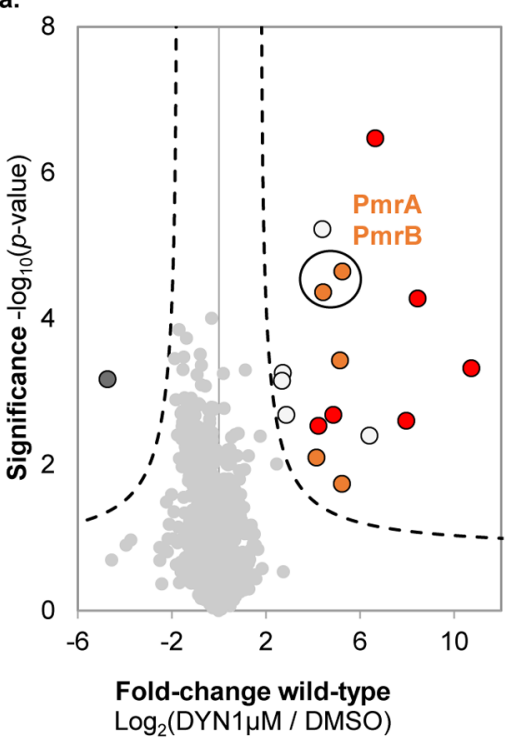

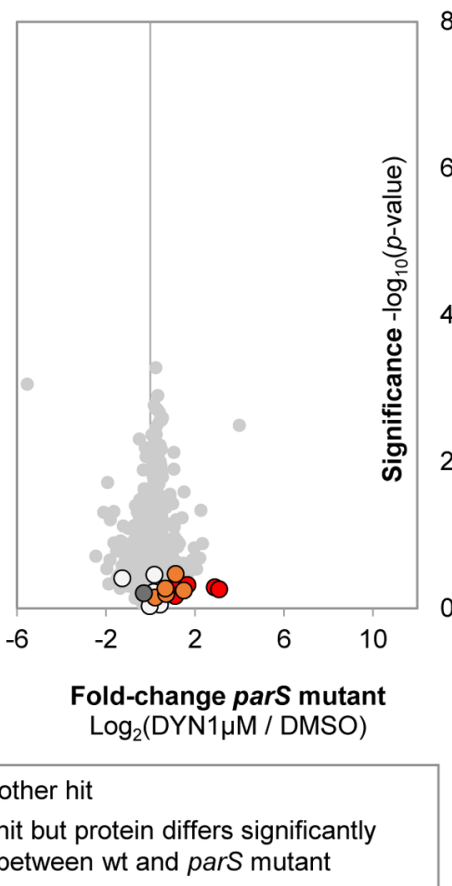

b. $\quad 1.2$

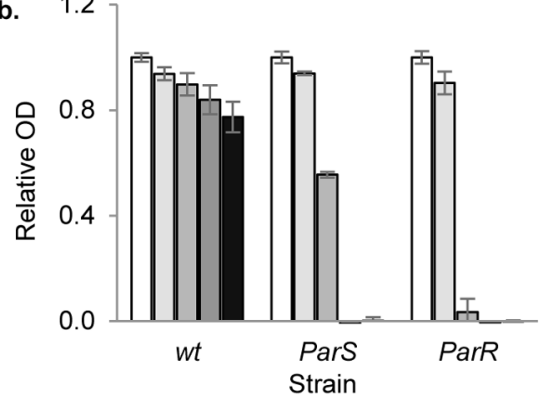

$\square 0 \mu \mathrm{M} \square 3 \mu \mathrm{M} \square 10 \mu \mathrm{M} \square 30 \mu \mathrm{M} \mathbf{\square} 50 \mu \mathrm{M}$

c.

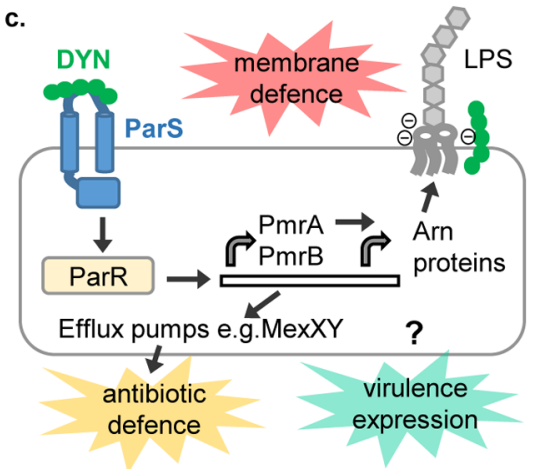

Figure 6. . ParS mediates the AMP-response induced by DYN treatment. (a) Volcano plot visualization of Stat.1 data set (wt or parS mutant bacteria grown for $16 \mathrm{~h}$ in the presence of $1 \mu \mathrm{M}$ DYN or DMSO control); $n=3$ replicates (grown from separate starter cultures on the same day). Bacteria were harvested, lysed, and samples prepared by FASP for LC-MS/MS. Quantification was carried out with MaxLFQ. After filtering to discard proteins not identified in at least two replicates, missing values were imputed (downshift 1.8, width 0.3 ), and a two-sample $t$-test (permutation-based, FDR 0.05 , s0 1) carried out to compare DMSO and DYN treated samples for each strain. No significant hits were obtained in the parS mutant, see also Table S5. The wt and mutant proteomes are compared in Figure S10. (b) parS and parR transposon mutants are more susceptible to treatment with DYN. Error bars show standard deviation from technical duplicates. Pyocyanin production in these mutants and others is shown in Figure S9. (c) Proposed model for DYN signaling via ParS to promote membrane defense mechanisms and induce virulence. DYN interacts with ParS sensor kinase, presumably triggering phosphorylation of the response regulator ParR and transcription of proteins in the pmrAB and arn operons. Proteins from these operons are involved in the antimicrobial peptide-liked response, specifically modifying the structure of LPS lipid A in the case of ArnT. Efflux pumps are also upregulated. Thus, ParS signaling triggers bacterial defense against antimicrobial peptides and antibiotics. The link to virulence expression (e.g., pyocyanin induction and upregulation of biofilm-related protein LecA) is yet to be elucidated.

PhoQ in Salmonella. ${ }^{35}$ The mechanism of mild bacterial growth inhibition by DYN also remains to be explored, but may be due to stress induced by membrane binding.

Treatment of $P A$ with Dyn-A has been previously linked to the PQS (Pseudomonas Quinolone Signal) quorum sensing (QS) system. ${ }^{4}$ AMPs, such as colistin, also induce expression of QS genes, ${ }^{36}$ and ParRS has been linked to modest changes in QS pathways in $P A .{ }^{37}$ However, we observed no upregulation of any QS proteins in our proteomic analyses. This could be due to the timing of analysis, or differences in the culture medium, which is a key determinate in the phenotypic response. Our proteomic data also do not readily explain the increase in pyocyanin production observed in response to DYN; of all the proteins involved in the synthesis of this toxin, only PhzB2 was upregulated. Again, this could reflect timing of analysis. How ParRS signaling may be linked to the circuitry for enhanced pyocyanin production is unclear, and it is possible that other sensors and pathways are involved in this aspect of the response. The other chemical proteomics hits identified here (Figure 4d; e.g., putative sensor/response regulator PA1243) are interesting starting points for further study.

We also observed DYN-induced upregulation of drug efflux pumps. A previous study showed that, unlike wt bacteria, a parRS mutant did not upregulate MexXY and downregulate the porin OprD in response to AMPs and that spontaneous mutants in parR and $S$ had heightened intrinsic resistance to diverse antibiotics. ${ }^{32}$ This may be clinically relevant since the AMP colistin and other antibiotics are given sequentially to cystic fibrosis patients. ${ }^{32,38}$ The present study thus adds to the increasing body of evidence linking virulence and antibiotic resistance in Pseudomonas and suggests that host factors can trigger a multiresistant phenotype in this pathogen.

\section{CONCLUSION}

In summary, we reveal that a human opioid peptide induces a specific defense mechanism in $P$. aeruginosa $(P A)$ via direct binding of the sensor ParS. We provide strong evidence that the antimicrobial peptide (AMP) response is mediated by ParS. Concentrations of up to $\sim 0.4 \mu \mathrm{M}$ Dyn-A have been reported in the $P A$-infected, injured gut ${ }^{4}$-in the same range as DYN concentrations that induced a AMP response-indicating that this effect could be biologically relevant in vivo. Our data thus supports the intriguing possibility that Pseudomonas can specifically recognize human stress hormones and initiate mechanisms to defend itself against the hostile environment of the host. This work highlights ParS as a promising drug target for blocking virulence induction and for sensitizing bacteria to antimicrobial therapies. 


\section{ASSOCIATED CONTENT}

\section{S Supporting Information}

The Supporting Information is available free of charge on the ACS Publications website at DOI: 10.1021/jacs.7b01072.

Table S1 (XLSX)

Table S2 (XLSX)

Table S3 (XLSX)

Table S4 (XLSX)

Table S5 (XLSX)

Detailed synthetic and experimental procedures, analytical (NMR, LCMS) characterization of compounds and peptides and Figures S1-S10 (PDF)

\section{AUTHOR INFORMATION}

\section{Corresponding Authors}

*stephan.sieber@mytum.de

*M.H.Wright@leeds.ac.uk

\section{ORCID}

Megan H. Wright: 0000-0003-2731-4707

Christian Fetzer: 0000-0001-5510-1544

Stephan A. Sieber: 0000-0002-9400-906X

\section{Present Address}

${ }^{\dagger}$ School of Chemistry, University of Leeds, Leeds, LS2 9JT, United Kingdom.

\section{Notes}

The authors declare no competing financial interest.

\section{ACKNOWLEDGMENTS}

We gratefully acknowledge funding from the European Union's Seventh Framework Programme under grant agreement PIEFGA-2013-625528 and the Center for Integrated Protein Science Munich (CIPSM). We thank the Manoil lab (University of Washington) for transposon mutants from the Pseudomonas aeruginosa PAO1 transposon mutant library (grant no. NIH P30 DK089507). We also thank Katja Bäuml and Nina Bach for mass spectrometry assistance, Mona Wolff for technical assistance, and Angela Weigert Muños, Mert Malay, and Thomas Löhr for synthetic assistance (internships). We thank Annabelle Hoegl and Matthias Stahl in the Sieber group for critical reading of this manuscript. Proteomics Datasets: The mass spectrometry proteomics data have been deposited to the ProteomeXchange Consortium via the PRIDE $^{39}$ partner repository with the data set identifier PXD006172.

\section{REFERENCES}

(1) Chavkin, C. Mol. Pharmacol. 2013, 83, 729.

(2) Sobczak, M.; Salaga, M.; Storr, M. A.; Fichna, J. J. Gastroenterol. 2014, 49, 24.

(3) Wu, L. R.; Zaborina, O.; Zaborin, A.; Chang, E. B.; Musch, M.; Holbrook, C.; Turner, J. R.; Alverdy, J. C. Surg Infect (Larchmt) 2005, 6, 185.

(4) Zaborina, O.; Lepine, F.; Xiao, G.; Valuckaite, V.; Chen, Y.; Li, T.; Ciancio, M.; Zaborin, A.; Petrof, E. O.; Turner, J. R.; Rahme, L. G.; Chang, E.; Alverdy, J. C. PLoS Pathog. 2007, 3, e35.

(5) Saiman, L. Paediatr Respir Rev. 2004, 5, S367.

(6) Balasubramanian, D.; Schneper, L.; Kumari, H.; Mathee, K. Nucleic Acids Res. 2013, 41, 1.

(7) Zaborin, A.; Gerdes, S.; Holbrook, C.; Liu, D. C.; Zaborina, O. Y.; Alverdy, J. C. PLoS One 2012, 7, e34883.

(8) Wu, L.; Estrada, O.; Zaborina, O.; Bains, M.; Shen, L.; Kohler, J. E.; Patel, N.; Musch, M. W.; Chang, E. B.; Fu, Y. X.; Jacobs, M. A.;
Nishimura, M. I.; Hancock, R. E.; Turner, J. R.; Alverdy, J. C. Science 2005, 309, 774

(9) Clarke, M. B.; Hughes, D. T.; Zhu, C.; Boedeker, E. C.; Sperandio, V. Proc. Natl. Acad. Sci. U. S. A. 2006, 103, 10420.

(10) Chavkin, C.; Goldstein, A. Proc. Natl. Acad. Sci. U. S. A. 1981, $78,6543$.

(11) Wright, M. H.; Sieber, S. A. Nat. Prod. Rep. 2016, 33, 681.

(12) Lapinsky, D. J. Bioorg. Med. Chem. 2012, 20, 6237.

(13) Geurink, P. P.; Prely, L. M.; van der Marel, G. A.; Bischoff, R.; Overkleeft, H. S. Top. Curr. Chem. 2011, 324, 85.

(14) Srinivas, N.; Jetter, P.; Ueberbacher, B. J.; Werneburg, M.; Zerbe, K.; Steinmann, J.; Van der Meijden, B.; Bernardini, F.; Lederer, A.; Dias, R. L.; Misson, P. E.; Henze, H.; Zumbrunn, J.; Gombert, F. O.; Obrecht, D.; Hunziker, P.; Schauer, S.; Ziegler, U.; Kach, A.; Eberl, L.; Riedel, K.; DeMarco, S. J.; Robinson, J. A. Science 2010, 327, 1010.

(15) Needham, B. D.; Trent, M. S. Nat. Rev. Microbiol. 2013, 11, 467.

(16) Boersema, P. J.; Raijmakers, R.; Lemeer, S.; Mohammed, S.; Heck, A. J. Nat. Protoc. 2009, 4, 484.

(17) Park, J.; Koh, M.; Koo, J. Y.; Lee, S.; Park, S. B. ACS Chem. Biol. 2016, 11, 44.

(18) Park, H.; Koo, J. Y.; Srikanth, Y. V.; Oh, S.; Lee, J.; Park, J.; Park, S. B. Chem. Commun. (Cambridge, U. K.) 2016, 52, 5828.

(19) Kleiner, P.; Heydenreuter, W.; Stahl, M.; Korotkov, V. S.; Sieber, S. A. Angew. Chem., Int. Ed. 2017, 56, 1396.

(20) Cox, J.; Hein, M. Y.; Luber, C. A.; Paron, I.; Nagaraj, N.; Mann, M. Mol. Cell. Proteomics 2014, 13, 2513.

(21) Hatzenpichler, R.; Scheller, S.; Tavormina, P. L.; Babin, B. M.; Tirrell, D. A.; Orphan, V. J. Environ. Microbiol. 2014, 16, 2568.

(22) Landgraf, P.; Antileo, E. R.; Schuman, E. M.; Dieterich, D. C. Methods Mol. Biol. 2015, 1266, 199.

(23) Winsor, G. L.; Griffiths, E. J.; Lo, R.; Dhillon, B. K.; Shay, J. A.; Brinkman, F. S. Nucleic Acids Res. 2016, 44, D646.

(24) Fernandez, L.; Gooderham, W. J.; Bains, M.; McPhee, J. B.; Wiegand, I.; Hancock, R. E. Antimicrob. Agents Chemother. 2010, 54, 3372.

(25) Brogden, K. A. Nat. Rev. Microbiol. 2005, 3, 238.

(26) Wisniewski, J. R.; Zougman, A.; Nagaraj, N.; Mann, M. Nat. Methods 2009, 6, 359.

(27) McPhee, J. B.; Lewenza, S.; Hancock, R. E. Mol. Microbiol. 2003, $50,205$.

(28) Fernandez, L.; Jenssen, H.; Bains, M.; Wiegand, I.; Gooderham, W. J.; Hancock, R. E. Antimicrob. Agents Chemother. 2012, 56, 6212.

(29) Johnson, L.; Mulcahy, H.; Kanevets, U.; Shi, Y.; Lewenza, S. J. Bacteriol. 2012, 194, 813.

(30) Vargiu, A. V.; Pos, K. M.; Poole, K.; Nikaido, H. Front. Microbiol. 2016, 7, 833 .

(31) Sun, J.; Deng, Z.; Yan, A. Biochem. Biophys. Res. Commun. 2014, 453, 254.

(32) Muller, C.; Plesiat, P.; Jeannot, K. Antimicrob. Agents Chemother. 2011, 55, 1211.

(33) Diggle, S. P.; Stacey, R. E.; Dodd, C.; Camara, M.; Williams, P.; Winzer, K. Environ. Microbiol. 2006, 8, 1095.

(34) Ernst, R. K.; Yi, E. C.; Guo, L.; Lim, K. B.; Burns, J. L.; Hackett, M.; Miller, S. I. Science 1999, 286, 1561.

(35) Bader, M. W.; Sanowar, S.; Daley, M. E.; Schneider, A. R.; Cho, U.; Xu, W.; Klevit, R. E.; Le Moual, H.; Miller, S. I. Cell 2005, 122, 461.

(36) Cummins, J.; Reen, F. J.; Baysse, C.; Mooij, M. J.; O’Gara, F. Microbiology 2009, 155, 2826.

(37) Wang, D.; Seeve, C.; Pierson, L. S., 3rd; Pierson, E. A. BMC Genomics 2013, 14, 618.

(38) Flume, P. A.; O’Sullivan, B. P.; Robinson, K. A.; Goss, C. H.; Mogayzel, P. J., Jr.; Willey-Courand, D. B.; Bujan, J.; Finder, J.; Lester, M.; Quittell, L.; Rosenblatt, R.; Vender, R. L.; Hazle, L.; Sabadosa, K.; Marshall, B.; Cystic Fibrosis Foundation, P. T. C.. Am. J. Respir. Crit. Care Med. 2007, 176, 957.

(39) Vizcaino, J. A.; Csordas, A.; Del-Toro, N.; Dianes, J. A.; Griss, J.; Lavidas, I.; Mayer, G.; Perez-Riverol, Y.; Reisinger, F.; Ternent, T.; Xu, Q. W.; Wang, R.; Hermjakob, H. Nucleic Acids Res. 2016, 44, 11033. 